\title{
PROTOCOLO PARA TRANSFERÊNCIA DE INFORMAÇÃO QUÂNTICA SEM TRANSMISSÃO DE PARTÍCULAS
}

Protocolo para transferência de informação quântica sem transmissão de partículas

Protocolo para transferir información cuántica sin transmisión de partículas

João Marcos Costa da Silva ${ }^{* 1}$, Matheus Pereira Lobo ${ }^{1}$

${ }^{1}$ LABID - Laboratório Interativo Digital, Licenciatura em Física, Universidade Federal do

Tocantins, Araguaína, Tocantins, Brasil.

*Correspondência: Universidade Federal do Tocantins, Campus Universitário de Araguaína, Curso de Licenciatura em Física. Av. Paraguai esq. com R. das Uxiramas s/n, Cimba, Araguaína, Tocantins, Brasil.CEP:77.824-838.e-mail: joaomarcoscosta7@gmail.com

Artigo recebido em 10/04//2020 aprovado em 03/11/2020 publicado em 06/11/2020.

\section{RESUMO}

Apresentamos a demonstração matemática e os respectivos conceitos para a construção de um protocolo para transferir um qubit desconhecido entre duas regiões distintas do espaço, com distâncias arbitrárias. Para isso, serão utilizadas portas quânticas (Hadamard, CNOT, X e Z), medições quânticas livres de interação e uma versão dual do efeito Zeno quântico. Mostramos que não são necessários canais clássicos de comunicação para a realização da transferência da informação quântica, como ocorre, por exemplo, no teleporte usual. Esse estudo é completamente teórico e contém demonstrações do fenômeno de contrafactualidade de forma detalhada, assim como os assuntos necessários para a construção do protocolo.

Palavras-chave: Medições livres de interação. Contrafactualidade. Portas Quânticas.

\section{ABSTRACT}

We present the mathematical demonstration and the respective concepts for the construction of a protocol to transfer an unknown qubit between two distinct regions of space, with arbitrary distances. For that, quantum gates (Hadamard, CNOT, X and Z), quantum interaction-free measurements and a dual version of the quantum Zeno effect will be used. We show that classic communication channels are not necessary to carry out the transfer of quantum information, as occurs, for example, in the usual teleportation. This study is completely theoretical and contains detailed demonstrations of the counterfactuality phenomenon, as well as the subjects necessary for the construction of the protocol.

Keywords: Interaction-free measurements. Counterfactuality. Quantum Gates.

\section{RESUMEN}

Presentamos la demostración matemática y los conceptos respectivos para la construcción de un protocolo para transferir un qubit desconocido entre dos regiones distintas del espacio, con distancias arbitrarias. Para eso, se utilizarán puertas cuánticas (Hadamard, CNOT, X y Z), mediciones libres de interacción cuántica y una versión dual del efecto cuántico Zeno. Mostramos que los canales de comunicación clásicos no son necesarios para llevar a cabo la transferencia de información cuántica, como ocurre, por ejemplo, en la teletransportación habitual. Este estudio es completamente teórico y contiene demostraciones detalladas del fenómeno de contrafactualidad, así como los temas necesarios para la construcción del protocolo.

Descriptores: Mediciones sin interacción. Contrafactualidad. Puertas Cuánticas. 


\section{INTRODUÇÃO}

O estudo da natureza nas pequenas escalas, em níveis atômicos e subatômicos, tornou-se um dos assuntos mais intrigantes da Física. Essa área recebeu o nome de Mecânica Quântica e para compreendê-la é necessário uma fundamentação teórica e matemática rebuscada.

Na Mecânica Quântica, há o que denominamos de Comunicação Quântica que envolve processos de Informação Quântica, como emaranhamento, não-localidade, teleporte quântico, dentre outros.

Dentre os fenômenos da Mecânica Quântica há a não-localidade, prevista em 1964 pelo físico John Bell [1] e provado experimentalmente, em 1982, por Alain Aspect [2]. A não-localidade vem como uma ferramenta de comunicação mais eficiente na execução de determinadas tarefas, já que o colapso ocorre acima da velocidade da luz.

Dentre as aplicações da não-localidade, podemos realizar a medida de um objeto para detectar sua existência em uma determinada região sem qualquer tipo de interação e não há a necessidade de saber informações prévias do objeto em estudo. Tal fenômeno é conhecido por "medições quânticas livres de interação" [3] e não há um análogo clássico que possa representá-lo.

O termo teleporte quântico foi usado pela primeira vez em 1993 por Charles Bennett e colaboradores [4] e teve sua comprovação experimental [5], que é realizado pelo emaranhamento e com a medição de uma das quatro bases de Bell,

$$
\begin{aligned}
& \left|\Psi^{ \pm}\right\rangle=\frac{1}{\sqrt{2}}(|01\rangle \pm|10\rangle), \\
& \left|\Phi^{ \pm}\right\rangle=\frac{1}{\sqrt{2}}(|00\rangle \pm|11\rangle) .
\end{aligned}
$$

E para isso são utilizadas três partículas sendo que uma delas viaja por um canal clássico de comunicação de uma região para outra [6].
Em contrapartida ao teleporte de Bennett et al, recentemente Salih [7] mostrou que é possível transportar um qubit desconhecido entre duas regiões distintas do espaço sem um canal clássico. Esse fenômeno é chamado por "contrafactualidade", utilizando ferramentas que já foram testadas experimentalmente - medições livres de interação e efeito Zeno quântico. Unindo esses dois fenômenos com algumas portas quânticas - CNOT, X, Z e Hadamard -, é possível a realização deste protocolo.

Apresentamos a demonstração matemática para a realização do protocolo contrafactual. Cada um dos tópicos supracitados será discutido de forma detalhada, de forma conceitual e matemática, para depois relacionar todos esses efeitos e chegar no protocolo de transferência de um qubit desconhecido contrafactualmente.

\section{MATERIAIS E MÉTODOS}

Este trabalho é puramente teórico; contempla estudos voltados aos Fundamentos da Mecânica Quântica, mais especificamente em Informação Quântica, por meio de livros especializados e artigos científicos. A bibliografia utilizada inclui textos didáticos e técnicos para compreensão dos assuntos necessários para, por fim, chegar no protocolo de contrafactualidade.

\section{RESULTADOS E DISCUSSÃO}

Para elaborar o protocolo de transmissão de informação quântica sem o envio de partículas entre uma região $A$ e uma região $B$, precisamos compreender medições livre de interações com interferômetro tipo Mach-Zehnder, efeito Zeno quântico e as portas lógicas quânticas supracitadas.

O interferômetro de Mach-Zehnder [3] utiliza um aparato experimental da óptica clássica, composto por dois espelhos (que refletem $100 \%$ da luz que incide, $M_{1}$ ), dois semi-espelhos (que refletem $50 \%$ da 
luz e refratam $50 \%, M_{2}$ ) e dois detectores, como ilustra a Figura 1.

Figura 1: Variante do Interferômetro de Mach-Zehnder [3]

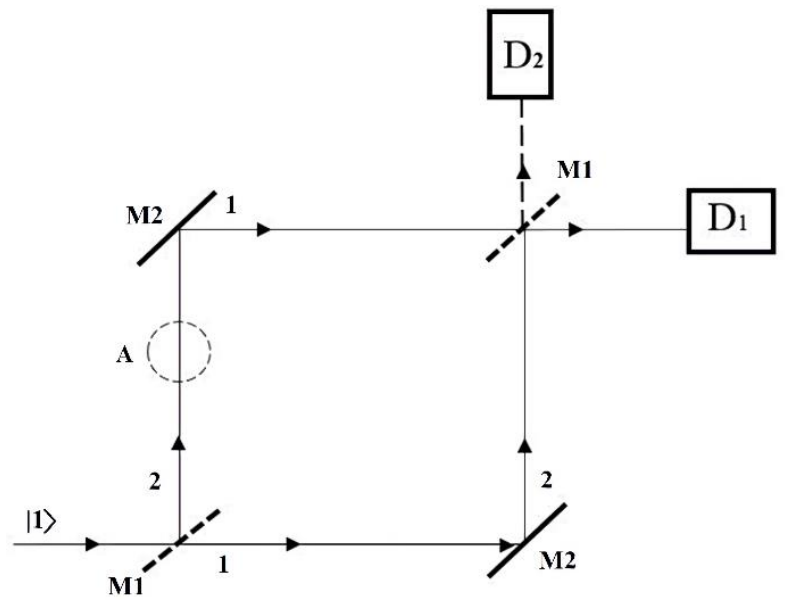

Assim, é possível utilizar um fóton para entrar em superposição de dois estados ao passar pelo primeiro espelho $M_{1}$, depois ser refletido pelos espelhos $M_{2}$ e, por fim, recombinado no segundo espelho $M_{1}$, seguindo em direção a um dos detectores, $D_{1}$ ou $D_{2}$.

Esse experimento pode ser realizado sem objeto obstruindo a passagem do fóton, com um objeto clássico ou com um objeto quântico. Quando não houver objeto em um dos percursos dos estados da função de onda, o $D_{1}$ clicará e quando houver um objeto obstruindo um dos caminhos, o $D_{2}$ clicará.

O Efeito Zeno Quântico (EZQ) teve sua origem em um clássico paradoxo lógico apresentado pelo filósofo Zenão de Eleia. O paradoxo pode ser explicado utilizando uma flecha voando através do espaço; e para intervalos de tempo determinados, não se pode dizer que a flecha está em movimento ou em repouso [8]. Hoje sabemos que há erros no raciocínio deste paradoxo.

As portas lógicas quânticas são circuitos que operam em qubits, isto é, são utilizadas para manipular qubits. É importante ressaltar que elas são reversíveis. $\mathrm{O}$ fato de serem reversíveis nos permite fazer manipulações em qubits sem perder informações, podendo refazer todo o processo de transformação no qubit.

A porta $X$ equivale à porta lógica clássica NOT e é capaz de fazer a seguinte transformação no estado inicial

$$
|0\rangle \leftrightarrow|1\rangle .
$$

A porta $Y$ equivale à rotação de $180^{\circ}$ no eixo y da esfera de Bloch e transforma os estados iniciais em

$$
\begin{aligned}
|0\rangle & \rightarrow i|1\rangle, \\
|1\rangle & \rightarrow i|0\rangle .
\end{aligned}
$$

A porta $Z$ equivale à rotação de $180^{\circ}$ no eixo $z$ da esfera de Bloch, mantendo o estado inicial $|0\rangle$ inalterado e mapeando em $|1\rangle$, então

$$
\begin{aligned}
|0\rangle & \rightarrow|0\rangle, \\
|1\rangle & \rightarrow-|1\rangle .
\end{aligned}
$$

Há, também, a porta Hadamard, que é capaz de produzir uma superposição e a sua medida associada tem amplitude de probabilidade de 50\% para se tornar 1 ou 0 , que realiza as seguintes transformações

$$
\begin{aligned}
& |0\rangle \rightarrow \frac{|0\rangle+|1\rangle}{\sqrt{2}}, \\
& |1\rangle \rightarrow \frac{|0\rangle-|1\rangle}{\sqrt{2}} .
\end{aligned}
$$

Já a porta CNOT possui dois qubits, um de controle e um alvo, e é capaz de produzir as transformações

$$
\begin{aligned}
|00\rangle & \rightarrow|00\rangle, \\
|01\rangle & \rightarrow|01\rangle, \\
|10\rangle & \rightarrow|11\rangle, \\
|11\rangle & \rightarrow|10\rangle .
\end{aligned}
$$

Note que, o primeiro qubit (controle) é responsável pela transformação do segundo qubit (alvo). Quando o qubit de controle é 0 , o alvo se mantém inalterado e quando é 1 , o alvo sofre uma transformação de seu estado quântico original. 
Assim, utilizamos apenas uma versão dual do canal do efeito Zeno quântico e as medições quânticas livres de interação para chegar a uma porta CNOT contrafactual e, por fim, para transferir um qubit desconhecido sem nenhuma partícula física viajando entre Alice e Bob.

Esse é um caso diferente do teleporte quântico convencional que utiliza um canal quântico e um clássico para a construção do protocolo de contrafactualidade. Aqui será apresentada toda a abordagem matemática e conceitual para chegar ao protocolo.

Figura 2: Mach-Zehnder CNOT contrafactual [7].
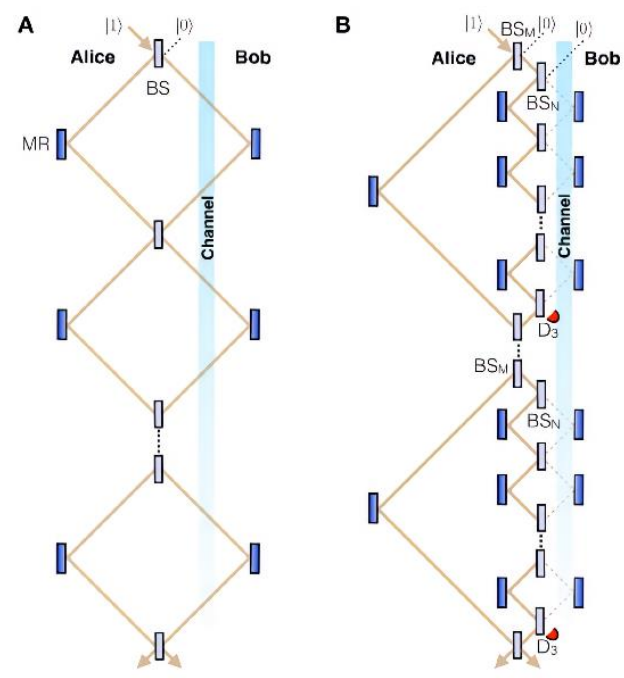

Levando-se em consideração a Figura 2A, o que devemos analisar, a priori, é a possibilidade de Bob estar ou não bloqueando o canal. Em termos práticos, trataremos $B S$ como espelhos. A ação de $B S$ no fóton de Alice é

$$
\begin{aligned}
& |10\rangle \rightarrow \cos \theta|10\rangle+\sin \theta|01\rangle, \\
& |01\rangle \rightarrow \cos \theta|01\rangle-\sin \theta|10\rangle,
\end{aligned}
$$

sendo o estado $|10\rangle$ à esquerda de BS e $|01\rangle$ à direita de BS, $\cos \theta=\sqrt{R}$ é a reflexão dos espelhos, $\theta=\frac{\pi}{2 N}$, e $N$ é o número de BS.

O estado combinado de Bob e Alice no primeiro espelho superior esquerdo é

$$
(\alpha \mid \text { pass }\rangle+\beta \mid \text { block }\rangle) \otimes|10\rangle,
$$

o estado |pass $\rangle$ se refere Bob não bloquear o canal e $\mid$ block $\rangle$ Bob bloqueando o canal, $\alpha$ e $\beta$ são as respectivas amplitudes de probabilidades.

$\mathrm{O}$ estado combinado é rotacionado depois do primeiro BS para

$$
(\alpha \mid \text { pass }\rangle+\beta \mid \text { block }\rangle) \otimes(\cos \theta|10\rangle+\sin \theta|01\rangle) .
$$

Antes do segundo BS, quando o objeto de Bob não bloqueia, temos o estado

$$
\begin{gathered}
\left(1-|\beta|^{2} \sin ^{2} \theta\right)^{\frac{1}{2}}[\alpha \mid \text { pass }\rangle \otimes(\cos \theta|01\rangle) \\
+\beta \mid \text { block }\rangle \otimes|10\rangle],
\end{gathered}
$$

com amplitude de probabilidade $\left(1-|\beta|^{2} \sin ^{2} \theta\right)$. Logo após o segundo BS,

$$
\begin{aligned}
& \left(1-|\beta|^{2} \sin ^{2} \theta\right)^{\frac{1}{2}}[\alpha \mid \text { pass }\rangle \otimes(\cos 2 \theta|10\rangle+ \\
& \sin 2 \theta|01\rangle)+\beta \mid \text { block }\rangle \otimes \cos \theta|10\rangle+\sin \theta|01\rangle] .
\end{aligned}
$$

Agora, vamos generalizar o sistema para $n \mathrm{BS}$,

$(\alpha \mid$ pass $\rangle+\beta \mid$ block $\rangle) \otimes|10\rangle \rightarrow$

$\left(-|\beta|^{2} \sin ^{2} \theta\right)^{\frac{(n-1)}{2}}[\alpha \mid$ pass $\rangle \otimes(\cos n \theta|10\rangle+$ $\sin n \theta|01\rangle)+\beta \mid$ block $\rangle \otimes \cos \theta|10\rangle+$ $\sin \theta|01\rangle]$,

se $N$ for muito grande, o estado combinando de Bob e Alice será

$$
(\alpha \mid \text { pass }\rangle+\beta \mid \text { block }\rangle) \otimes|10\rangle,
$$

com amplitude de probabilidade dada por 1 $|\beta|^{2} \sin ^{2} \theta^{\frac{(n-1)}{2}}$.

Obtemos, assim, uma porta CNOT com Bob como o controle do qubit,

$$
\begin{gathered}
\mid \text { block }\rangle \equiv|0\rangle, \\
\mid \text { pass }\rangle \equiv|1\rangle,
\end{gathered}
$$

e Alice como o qubit alvo,

$$
\begin{aligned}
|10\rangle & \equiv|0\rangle, \\
|01\rangle & \equiv|1\rangle,
\end{aligned}
$$

mesmo que seja possível apenas um dos possíveis estados de Alice, $|0\rangle$.

Quando a superposição corresponde ao objeto de Bob bloqueando o canal, podemos dizer que é contrafactual, e quando não bloqueia não é contrafactual.

Para efetuar completamente o CNOT contrafactual, usamos o canal do efeito Zeno quântico Revista Desafios -v. 7, Especial - PIBIC, 2020 
ilustrado na Fig. 2A. O fóton de Alice viaja entre os $M$ BS, ou $B S_{M}, \operatorname{com} \theta=\frac{\pi}{2 M}$, depois o fóton viaja entre os N BS, ou $B S_{N}, \operatorname{com} \theta=\frac{\pi}{2 N}$, assumindo os estados $|100\rangle$ quando estiver à esquerda de $B S_{M},|010\rangle$ à direita de $B S_{M}$ e à esquerda de $B S_{N}$ e $|001\rangle$ à direita de $B S_{N}$. Assim, depois de $m$ ciclos, a partir da eq. (2), obtemos

$$
\begin{gathered}
(\alpha \mid \text { pass }\rangle+\beta \mid \text { block }\rangle) \otimes|010\rangle \rightarrow \\
\left(1-|\beta|^{2} \sin ^{2} \theta\right)^{\frac{(n-1)}{2}}[\alpha \mid \text { pass }\rangle \otimes\left(\cos n \theta_{N}|010\rangle+\right. \\
\left.\left.\sin n \theta_{N}|001\rangle\right)+\beta \mid \text { block }\right\rangle \otimes \cos \theta_{N}|010\rangle+ \\
\left.\sin \theta_{N}|001\rangle\right],
\end{gathered}
$$

depois de $n B S_{N}$, com $n$ muito grande, o estado de Alice e Bob é

$$
(\alpha \mid \text { pass }\rangle|001\rangle+\beta \mid \text { block }\rangle|010\rangle),
$$

mantendo a amplitude de probabilidade 1 $|\beta|^{2} \sin ^{2} \theta^{\frac{(n-1)}{2}}$.

Um único fóton de Alice tem o estado inicial $|100\rangle$ (Fig. 2B); depois de $m B S_{M}$, temos

$$
\begin{array}{r}
(\alpha \mid \text { pass }\rangle+\beta \mid \text { block }\rangle) \otimes|100\rangle \rightarrow \\
\left(1-|\beta|^{2} \sin ^{2} \theta\right)^{\frac{(m-1)}{2}}[\alpha \mid \text { pass }\rangle \otimes \\
\left.\left(\cos n \theta_{M}|100\rangle+\sin n \theta_{M}|010\rangle\right)+\beta \mid \text { block }\right\rangle \otimes \\
\left.\cos \theta_{M}|100\rangle+\quad \sin \theta_{M}|010\rangle\right] ;
\end{array}
$$

depois de $m B S_{M}$, com $n$ muito grande, o estado de Alice e Bob é

$$
\approx(\alpha \mid \text { pass }\rangle|100\rangle+\beta \mid \text { block }\rangle|010\rangle),
$$

mantendo a amplitude de probabilidade 1 $|\beta|^{2} \sin ^{2} \theta^{\frac{(m-1)}{2}}$.

Finalmente, temos as ferramentas para construir o protocolo contrafactual para teleportar um qubit desconhecido. Para isso, utilizaremos duas portas CNOT (Fig. 3A) com estados de entrada $(\alpha|0\rangle+\beta|1\rangle)$ se convertendo em $|0\rangle$ na parte superior e transferindo para a inferior. Aplicando a rede da Fig. 3A na rede da Fig. 3C, obtemos a base para o protocolo,

$$
(\alpha|0\rangle+\beta|1\rangle) \otimes|0\rangle \stackrel{\text { CNOT }}{\longrightarrow}
$$

$$
\begin{gathered}
\alpha|00\rangle+\beta|11\rangle \stackrel{H^{\otimes 2}}{\longrightarrow} \\
\alpha|++\rangle+\beta|--\rangle \stackrel{\text { CNOT }}{\longrightarrow} \\
\alpha|++\rangle+\beta|+-\rangle \stackrel{H^{\otimes 2}}{\longrightarrow} \\
|0\rangle \otimes(\alpha|0\rangle+\beta|1\rangle) .
\end{gathered}
$$

Unindo o resultado de (5) na rede da Fig. 3C, iniciamos a rede da Figura 3D. Assim, aplicando as portas X e Z, obtemos

$$
\begin{gathered}
(\alpha|0\rangle+\beta|1\rangle) \otimes|0\rangle \stackrel{\text { CNOT }}{\longrightarrow} \\
\alpha|00\rangle+\beta|11\rangle \stackrel{H^{\otimes 2}}{\longrightarrow} \\
\alpha|++\rangle+\beta|--\rangle \stackrel{I \otimes Z}{\longrightarrow} \\
\alpha|+-\rangle+\beta|-+\rangle \stackrel{C N O T}{\longrightarrow} \\
\alpha|--\rangle+\beta|-+\rangle \stackrel{H^{\otimes 2}}{\longrightarrow} \\
\alpha|11\rangle+\beta|10\rangle \stackrel{I \otimes X}{\longrightarrow} \\
|1\rangle \otimes(\alpha|0\rangle+\beta|1\rangle) .
\end{gathered}
$$

Finalmente, obtemos, assim, o protocolo para transferir o qubit de Bob contrafactualmente para Alice.

Figura 3: Rede de transferência de estado quântico [4].
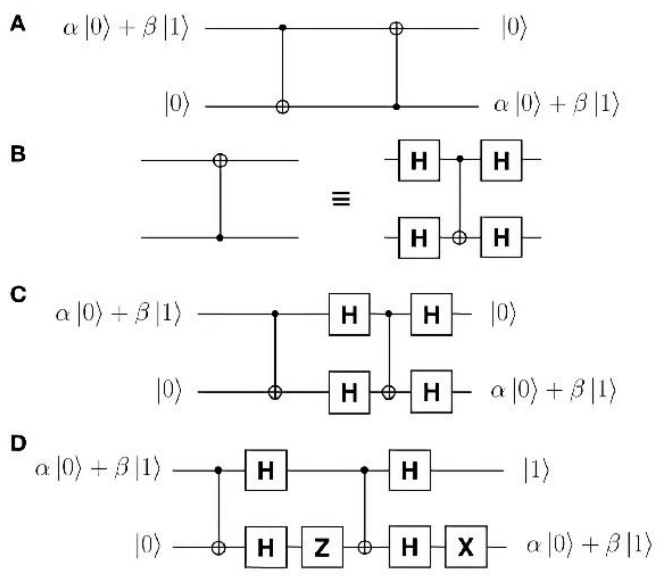

\section{CONCLUSÃO}

Desde o início da Mecânica Quântica, as formulações matemáticas se tornaram cada vez mais robustas para acompanhar as diversas interpretações conceituais que não existem no mundo clássico. Em virtude disso, o estudo dela tem ganhado mais força à medida que as pesquisas avançam.

De fato, mostramos que é possível transferir informações entre duas regiões do espaço via Revista Desafios -v. 7, Especial - PIBIC, 2020 
contrafactualidade, isto é, SEM a transmissão de partículas a partir dos resultados da teoria da Informação Quântica que já conhecemos. Esse tipo de resultado abre novas formas de interpretação da natureza quântica e abre espaço para aplicações a curto e médio prazo, já que sua formulação matemática e comprovação experimental são suficientemente robustas.

Por isso, este trabalho explicou detalhadamente todo o processo da elaboração do mesmo para que estudantes possam compreender por completo a importância dos processos envolvidos na comunicação quântica dentro do contexto da Informação Quântica.

\section{AGRADECIMENTO}

Agradecemos ao $\mathrm{CNPq}$ e à Universidade Federal do Tocantins pelo suporte necessário para a realização deste trabalho. Agradecemos também, ao LABID - Laboratório Interativo Digital - pelo acolhimento e tornar este trabalho possível.

\section{REFERÊNCIAS}

[1] BELL, J. S. Physica 1, 195 (1964).

[2] ASPECT, A.; GRANGIER, P.; ROGER, G. Physical Review Letters 49 (1982).

[3] ELITZUR, A. C.; VAIDMAN, L. Quantum Mechanical Interaction-Free Measurements. Foundations of Physics 23, 987 (1993).

[4] MIRANOWICZ, A.; TAMAKI, K. An Introduction to Quantum Teleportation. Math. Sciences (Suri-Kagaku) 473, 28 (2002).

[5] BOUWMEESTER D.; PAN, J.; MATTLE, K.; EIBL, M.; WEINFURTER, H.; ZEILINGER, A. Nature 390 (1997).

[6] LOBO, M. P.; GOMES, S. L. R.; ALENCAR, E. A.; SANTOS, C. M. F. Teleporte de uma Partícula: um Protocolo no Contexto do Ensino Médio. Física na Escola, 26, p. 18-21 (2018).

[7] SALIH, H. Protocol for Counterfactually Transporting an Unknown Qubit. Front. Phys. 3, 9 (2014).

[8] AL-KHALILI. Paradox: the mine greatest Enigmas in Physics. Band 1, New York: Broadway Paperbacks, (2012) 